\title{
L'informazione sul libro: miraggi e prospettive della comunicazione multimediale $^{1}$
}

Per affrontare il tema della comunicazione libraria nella società multimediale è necessario seguire, sia pure in sintesi, le sorti dei protagonisti indiscussi di questa operazione informativa: l'autore, l'opera e il pubblico. Attori di una rappresentazione che ha variamente mutato il proprio copione nel corso della storia culturale dell'Occidente (dalla prima rivoluzione, l'invenzione della stampa, alla terza cui assistiamo in tempo reale, la trasmissione elettronica del testo, passando attraverso il processo di industrializzazione del libro stampato, che rappresenta il secondo cataclisma per l'editoria occidentale), essi sono realmente i punti fermi di un gioco complesso di richiami e di sollecitazioni linguistiche (verbali e non), che ormai di regola corredano l'ingresso e il cammino di ogni tipo di libro 'sul mercato'.

Presento qui alcune riflessioni relative ai mutamenti in fieri che il libro subisce nelle "catene di trasmissione', ${ }^{2}$ per dirla con Doležel (1990), ed alle ripercussioni sostanziali che i modi di leggere e di informare il pubblico sulla lettura porteranno (è lecito, quanto meno, sospettarlo) sui criteri di codificazione testuale.

Il quesito di fondo può essere riassunto nel modo seguente: l'attività di scrittura finirà per essere condizionata 0 , addirittura, trasformata nella sua natura intrinseca (un esercizio individuale, indirizzato ad un destinatario assente), ad opera delle nuove tecniche di ricezione del libro da parte del lettore e dei differenti modi di presentare e di informare il lettore sulle qualità del libro da parte dell'editore? Scrittura e lettura, in altre parole, stanno forse perdendo le loro sembianze tradizionali, per rinascere in altre vesti nel contesto della comunicazione multimediale, non trovando in esso gli spazi che la civiltà dell'oralità e, successivamente, quella della scrittura avevano loro assegnato dall 'antichità ai giorni nostri?

Il tema è affascinante quanto complesso e nei limiti qui dettati dal tempo sarà possibile esplicitare soltanto alcuni dei molti spunti teorici che ne derivano: le dimensioni sociale ed economica, egualmente coinvolte nell'argomento discusso in questo seminario, saranno volutamente trascurate in queste pagine, a beneficio degli aspetti semiotici e linguistici. 
Dell 'importanza che il momento della ricezione riveste nell'atto comunicativo che ciascun testo significa erano ben consapevoli gli intellettuali del Circolo linguistico di Praga, iniziatori, fra le altre cose, di quel filone di studi che si concretizzerà nella 'poetica semiotica' e nella 'estetica della ricezione'. ${ }^{3}$ In particolare, il progetto praghese aveva inaugurato la considerazione degli aspetti esterni della comunicazione letteraria: i rapporti fra produttori e letteratura e quelli, ancora più sfuggenti, fra letteratura e pubblico. La definizione del testo come oggetto semiotico nasce in quell'ambiente in stretto riferimento all'opera letteraria, ma risulta estensibile, nella sostanza, anche ad altri generi (saggistica, autobiografia etc.). Lo scritto, ricorda Doležel, fa parte di un circuito comunicativo segmentato in due tranches, in conformità al canale diamesico a cui appartiene: mittente (autore)-messaggio e messaggio-ricevente (pubblico). I due poli del circuito non si incontrano e il trait d'union che li collega resta la pagina scritta, compilata e ordinata in una sequenza immutabile, su cui il destinatario non può compiere interventi che ne modifichino fisicamente la struttura. La fissità della parola scritta è infatti tradizionalmente contrapposta al dinamismo camaleontico del parlato, come sua proprietà specifica e caratterizzante. ${ }^{4}$ Sono però possibili altri tipi di interazione fra pubblico dei lettori e testo: l'insieme delle operazioni interpretative che ogni epoca variamente assegna alle singole opere, ai libri che ne rappresentano il canone garantisce a ciascun prodotto letterario la propria sopravvivenza nel tempo e nella storia (all'interno, appunto, delle cosiddette "catene di trasmissione') e suggellano il compimento del ciclo comunicativo. ${ }^{5}$ Si può, pertanto, affermare che un testo ha raggiunto i fini comunicativi per cui è stato generato, quando il pubblico-destinatario l'ha reinterpretato ed accolto. Doležel propone per questa fase rielaborativa ad opera del lettore la definizione di 'transduzione letteraria' [literacy transduction] (210). L'espressione intende cogliere, negli intenti di chi l'ha coniata, il trasformismo filologico, quel continuo divenire cui ogni forma di testualità letteraria (e non) soggiace, quando il libro intraprende i percorsi diasporici della diffusione e della distribuzione commerciale.

Il lettore disporrà di uno spazio creativo maggiore o minore, a seconda delle strategie di codificazione e in base alle tecniche che consentono ad un insieme di significati rappresentati e prodotti (secondo nuove modalità definitorie suggerite oltroceano nell'ambito dei cultural studies, per la nozione di 'testo' $)^{6}$ di superare il momento e il luogo della stesura originaria. La 'transduzione' varia perciò in diacronia, poiché cambiano nel tempo i mezzi di articolazione linguistica del messaggio e di confezione esterna del libro (che ne rappresenta, in qualche misura, il 'contenitore commerciale'), ma varia anche in prospettiva sincronica, dato che agli strumenti storicamente impiegati per la scrittura e la lettura-ricezione (rispettivamente: la penna o la macchina da scrivere, la consegna del manoscritto all 'editore che provvede alla stampa e la lettura individuale del materiale cartaceo) si affiancano ormai 
le procedure informatiche e multimediali. Il testo è sempre più spesso composto e memorizzato con programmi di scrittura (word processors) su personal computer e con frequenza crescente le case editrici, per quanto prestigiose e ben presenti sul mercato nazionale ed internazionale, incoraggiano l'autore a curare personalmente l'editing. Questo passaggio di mansioni porta due tipi di conseguenze di segno opposto. ma di eguale portata: sul piano economico, l'editing 'fatto in casa' si traduce nell'abbattimento dei costi di produzione; d'altro lato, sottraendo le attività di preparazione formale ed estetica del libro al diretto controllo dei tecnici editoriali (impaginazione, scelta dei caratteri etc.) si rischia di compromettere fortemente la qualita finale del prodotto. In altre parole, la migrazione della perizia professionale necessaria a redigere libri di qualità dalla casa editrice al tavolo (o forse meglio. alla tastiera) dell'autore non pud avvenire senza un progressivo detrimento delle competenze redazionali.?

L'atto terminale del processo di trasmissione è la lettura. La 'rivoluzione multimediale` finirà per investire anche questa fase della circolazione libraria, più di quanto non sia avvenuto finora. La produzione di opere in $C D-R o m$ prevede, infatti, che anche il lettore, passando dalla passività ricettiva imposta dalla versione a stampa alla mobilità dello scorrimento a video, interagisca attivamente col testo. Ad uno stile di lettura lineare si va sostituendo un andamento desultorio ed interattivo.

Il circuito comunicativo di cui il libro fa parte come oggetto semiotico (ossia veicolo di un messaggio rivolto da un emittente ad una pluralità indefinita di riceventi) è costituito anche da un insieme di fattori esterni: essi rappresentano, nella loro totalità, il corredo informativo che la moderna politica editoriale associa ormai sistematicamente al lancio dei propri prodotti librari sul mercato. Fattori comunicativi interni e dati informativi esterni hanno ripercussioni non trascurabili sulla vita e sulla vitalita del libro nella comunità scientifica e letteraria cui è destinato; una rapida rassegna degli elementi ascrivibili alle due categorie permetterà di ricostruire gran parte delle trasformazioni del processo comunicativo di cui il libro è soggetto e oggetto al tempo stesso, come veicolo di cultura e come strumento di ricezione del messaggio.

Esaminando l'evoluzione dei fattori interni, si devono menzionare i ribaltamenti paradigmatici che hanno segnato la storia del libro. Per questo punto, faccio implicito riferimento alla recente miscellanea curata da Cavallo e Chartier, che indicano le seguenti tappe:

1. il mondo antico, dove il libro si presentava sotto forma di rotolo e il cui contenuto era destinato, per lo più, ad una lettura recitata e collettiva. Basti pensare alla trasmissione dell'epos omerico, filtrato e adattato di volta in volta alle esigenze dell'uditorio dalla collegialità rapsodica, attraverso un esercizio mnemonico fatalmente votato ad una repentina scomparsa. La scrit- 
tura in questo caso è, per molti versi, assimilabile a ciò che in epoca moderna definiamo ghost writing: se il nome dell autore resta a suggello di garanzia della qualità e continua a rappresentare il responsabile putativo del testo $(\mathrm{Ge}-$ nette), non sembra troppo rilevante l'identificazione del chi abbia provveduto alla stesura dell'opera in prima persona. né di quanti vi abbiano eventualmente partecipato oltre all'autore cui si attribuisce la paternità primaria. Altrettanto spersonalizzata è l'attività complementare della lettura, che costituisce uno dei momenti di massima aggregazione culturale della comunità. Siamo ancora lontani dall'atmosfera ieratica del monastero medioevale, in cui il rapporto lettore/testo si consolida nella dimensione individuale;

2. in epoca tardo-latina (con una tendenza che troverà ampia realizzazione nell'alto medioevo), si passa dalla lettura ad alta voce alla lettura silenziosa o mormorata: ${ }^{8}$ il libro, nel frattempo, ha assunto l'effigie più moderna del codice, vicina per alcuni tratti al formato dei nostri giorni. Le pagine vengono sfogliate sequenzialmente e ripropongono al lettore l'ordine lineare voluto dall'autore, o da chi ha restituito filologicamente il testo, suggerendogli un percorso prestabilito di lettura. Lo spazio per interventi interpretativi, quali testimonianze dell'avvenuto processo di ricezione, sono confinati alle annotazioni in margine alle colonne di cui è composta la pagina o alle eventuali osservazioni riportate inter lineas. Il margine creativo, cui facevamo cenno sopra, si è assottigliato, così come le possibilità di trasformazione della versione testuale primaria. Il passaggio dalla civiltà aurale alla civiltà della scrittura (tale è da considerarsi l'epoca che inizia nel tardo impero) avvicina per un verso autore e lettore, ne personalizza il rapporto, ma riduce la creatività ricettiva del pubblico;

3. non altrettanto carica di conseguenze, per quanto strano possa sembrare, è stata l'invenzione della stampa: le trasformazioni subite dal libro in questa fase storica sono pesanti sul piano grafico ed estetico, ma non così rilevanti su quello della sua trasmissione. Tratti di continuità più che elementi di rottura accomunano print culture e scribal culture: già il libro a codice si era, infatti, dotato di versioni in qualche modo 'tascabili' (si pensi al libellus di cui fa esplicita menzione, non senza qualche civetteria, Catullo, nell'incipit dedicatorio a Cornelio Gallo). E, d'altro lato, la tecnica di lettura silenziosa e visuale avviata in pieno alto medioevo mantiene le stesse caratteristiche in epoca moderna;

4. nihil novum sub sole, quindi, almeno fino al momento della messa in produzione su larga scala del libro a stampa (XVIII sec.), primo significativo passo verso un allargamento quantitativo dei potenziali destinatari. Cambia, in questa fase, anche il profilo qualitativo del lettore: al cosiddetto lettore intensivo (identificabile in prima istanza col clericus e culturalmente dipendente, comunque, dalla formazione religiosa) comincia ad affiancarsi un tipo di lettura laica, in senso lato, nasce cioè quella curiositas intellettuale che spingerà il pubblico degli alfabetizzati, per quanto ancora molto ristretto in 
termini numerici, a superare i confini del genere sacrale, alla ricerca quasi rabbiosa (si parla, infatti, di Lesewut "furore di leggere") di nuovi orizzonti testuali. La Pamela di Richardson (1740) incontra in questo periodo un inatteso successo di pubblico (soprattutto femminile) ed apre il varco ad un genere di romanzo più vicino alla concretezza della vita quotidiana (Wittmann);

5. l'ultima delle rivoluzioni di cui parlano Cavallo e Chartier è quella tuttora in corso: ne sono coinvolti in massima parte l'autore, che torna ad essere figura meno rigidamente fissata di quanto non capitasse con la civiltà della scrittura tradizionale (si pensi solo ai possibili interventi su dischetto dei molteplici revisori di un testo compiuterizzato) e il lettore, infine, senz'altro più libero di interagire con la parola scritta a video e, quel che più conta, autorizzato a spostarsi liberamente nel testo, che risulta ormai violato nel principio della sequenzialità lineare caratteristico della stampa.

Ciò implica, di necessità, che il progetto testuale concepito dall'autore in origine possa essere infranto legittimamente dal lettore più e più volte, senza che venga meno, per questo motivo, l'integrità dell'opera. Il testo ideato e trasmesso per via diatelica è strutturato per associazione di sezioni monografiche disposte in parallelo piuttosto che in serie. ${ }^{9}$ Questo tipo di innovazione interna ha portato le conseguenze più incisive sulla natura del circuito comunicativo che il libro percorre nella civiltà multimediale. Alcuni aspetti restano nell'ombra ed è difficile prevederne gli sviluppi: si pensi, per esempio, al problema della distinzione di ruoli e funzioni fra scrittore e lettore, non sempre così nettamente separabili nella comunicazione per via informatica; ${ }^{10}$ oppure alla questione della differenza qualitativa fra cultura mediale (con costi di produzione molto inferiori e conseguente abbassamento del livello qualitativo) e cultura tradizionale (di alto livello ed ancora legata agli strumenti di trasmissione ereditati dal passato recente). Negli Stati Uniti la dicotomia si sta già realizzando, con gravi rischi per gli equilibri culturali e sociali di quel paese.

Resta da prendere in esame i fattori di ordine esterno: essi includono il pacchetto informativo che in epoca moderna accompagna il testo dall'editore al mercato. I cambiamenti intervenuti a questo livello sono globalmente riconducibili ad un'unica generale tendenza: l'attenzione si è andata progressivamente spostando dal testo all'autore, come responsabile e garante della credibilità dei contenuti agli occhi del destinatario e, sul versante delle aspettative nutrite in casa editrice, della vendibilità dell'opera.

La storia dell 'informazione sul libro è anche storia delle pratiche di titolatura e di attribuzione di una paternità specifica alle singole opere. Genette ha chiaramente dimostrato come da una cultura fondata su una pratica di trasmissione pressoché anonima dei testi classici si sia passati alla necessaria indicazione del nome dell'autore nella civiltà libraria moderna. Anticamente, è noto, il nome dell'autore veniva inframezzato al testo (spesso nell'incipit o 
nell'explicit); Erodoto, per esempio, inserisce il proprio nome come prima parola delle Storie: Érodótou Alikarnasséōs istoríês apódexis hédé" "questa è l'esposizione delle ricerche di Erodoto di Alicarnasso". In altri casi, la paternità doveva essere attribuita postuma, in forma indiretta (è il noto e controverso caso di Omero). In epoca moderna non solo l'autore tiene a suggellare il testo con la propria sphrëgís, ma la convalida derivante dalla consuetudine autografa è richiamata con insistenza, tanto da tradursi in vera e propria forza persuasiva.

Esaminiamo più in dettaglio le strategie di comunicazione esterna. La sfera in cui di solito confluiscono le informazioni promozionali, o di natura pubblicitaria, è quella che Genette definisce paratesto, cioè "l'insieme eteroclito di pratiche e di discorsi di tutti i tipi e di tutte le età", che accompagnano la presentazione di ogni opera letteraria (Genette 4). ${ }^{11}$ Attraverso il paratesto un testo diventa libro e in quanto tale si propone ai lettori e al pubblico. Alcuni esempi: il titolo, l'indicazione del nome dell'autore, la prefazione, le note e, inoltre, tutto cio che produce informazione al di fuori dei confini della pagina scritta (interviste, dibattiti, incontri etc.). Le notizie dirette sono rafforzate dall'insieme fattuale di eventi che, se comunicati al pubblico, condizionano e modificano la ricezione dei contenuti: le vicende private relative alla vita dell'autore, $\mathrm{i}$ tratti della sua personalita etc. Conoscere o ignorare l'omosessualità di Proust, ci ricorda Genette, differenzia il modo in cui il messaggio della Recherche viene accolto. E tanti altri esempi dello stesso genere potrebbero essere elencati a sostegno dell'ipotesi per cui la biografia dell'autore esercita un condizionamento concreto sul secondo segmento del circuito comunicativo: il rapporto testo-pubblico.

La nostra cultura editoriale e il canale ricettivo comunemente diffuso nei paesi occidentali ha notevolmente potenziato gli elementi costitutivi del paratesto e fa ampio uso, a scopi di promozione e vendita, del cosiddetto paratesto 'fattuale'. ${ }^{12}$ Per quanto sia difficile misurarne il peso, ogni elemento biografico o aneddotico costituisce la cornice del testo, da cui il lettore non può prescindere nel valutarlo anche sotto il profilo estetico. ${ }^{13}$ Il messaggio, verbale e visivo, espresso in questa forma risponde a precise intenzioni e interpretazioni autoriali e editoriali. Pur trattandosi di discorso eteronomo, ausiliare, che si pone al servizio del testo, esso ha finito per rappresentarne la sua ragione d'essere.

Le parti di cui si compone fisicamente il libro seguono storicamente lo sviluppo del mercato dell'editoria nelle singole epoche. Dettagli apparentemente trascurabili, come i cambiamenti della copertina nel corso del '900 e la trasformazione, non ancora cessata, della tipologia dei titoli, illustrano, pertanto, le scelte strategiche dell'editoria sul piano commerciale e comunicativo. Ancora da Genette ( $26 \mathrm{sgg}$.) attingiamo preziose notizie sul mutare dei 'dintorni del testo' nel tempo. La copertina, è noto, nasce come luogo strategico per l'ideazione di inserti pubblicitari ante litteram, agli inizi dell'800: 
Genette cita, fra i primi esemplari, le Oeuvres complètes di Voltaire presso Baudoin (1825). A partire da quel momento, la veste anonima e dimessa caratteristica del libro antico riaffiora ormai solo occasionalmente sui banchi di vendita delle librerie antiquarie. In epoca moderna e, soprattutto, negli ultimi decenni la copertina si è arricchita di messaggi affidati al linguaggio o all'immagine, il cui significato non si esaurisce nella sfera referenziale e descrittiva; i tratti informativi più enfatici sono, infatti, concentrati in questa sezione (illustrazioni vistose, citazione di adattamenti cinematografici o televisivi etc.). Ad essa si affianca con frequenza crescente la cosiddetta sovracoperta (liseuse), dove trovano sede, di preferenza, le indicazioni iconografiche. In sovracoperta possiamo vedere la fotografia di un dipinto che richiama il contenuto, per una o più proprietà: il saggio sulla storia della lettura, per esempio, curato da Cavallo e Chartier reca in sovracoperta una foto del celebre quadro di Pablo Picasso dal titolo La lettrice. In tale modo, l'attenzione del lettore (o del potenziale acquirente) viene orientata direttamente verso il tema dell'opera. La scelta di un dipinto famoso, o, comunque, semanticamente attinente al Leitmotiv del libro sembra essere la più comune e, probabilmente, la più efficace in termini promozionali, poiché decodificare il messaggio visivo, in abbinamento a quello verbale condensato nel titolo, risulta operazione facile e immediata per il lettore. Qualche volta, il rapporto semantico fra l'indicazione iconografica e quella verbale non è di natura analogica, ma metonimica: il volume di M. Kundera (1995) La lentezza, pubblicato da Adelphi, ha impresso in un riquadro che copre circa la metà superiore della sovracoperta/copertina ${ }^{14}$ la tela del Tiepolo Passeggiata in villa. Il tema della lentezza (che nella matematica esistenziale di Kundera assume valore categoriale) è citato indirettamente attraverso la memoria del tema contiguo del quieto passeggiare in campagna, caro all'aristocrazia settecentesca raffigurata dal Tiepolo.

Altre volte, la sovracoperta ( $o$, in sua assenza, la copertina), contiene la foto dell'autore; questa linea in Italia è stata scelta soprattutto da quegli editori che privilegiano la stampa di volumi destinati al grande pubblico (da Mondadori a Rizzoli, o, in circostanze occasionali, Adelphi). ${ }^{15}$ La figura dell'autore sovrasta il profilo contenutistico dell'opera, come dire che la promozione è affidata prevalentemente alla sua immagine personale e alla presa che essa esercita sul potenziale acquirente. Anche questo è un sintomo del mutato rapporto fra autore e testo: la trasformazione della veste editoriale appare mossa complessivamente dalla tendenza al potenziamento del paratesto, ivi incluso tutto il materiale pubblicitario che di solito accompagna l'esposizione del volume in libreria (manifesti, stralci di recensioni etc.).

Il titolo resta depositario di una importante funzione 'adescatrice' nei confronti del pubblico dei lettori (un bel titolo è il vero prosseneta del libro, ci ricorda con qualche malizia, Genette) e, in obbedienza a tale compito, si sono incrementati e raffinati, nel tempo, i tratti di espressività linguistica. L'infor- 
mazione di natura referenziale viene rimandata al sottotitolo, il cui valore oscilla spesso fra l'epesegesi e il richiamo deittico. ${ }^{16} \mathrm{~L}$ 'espressività può essere ottenuta attraverso una serie di strumenti linguistici o retorici: fra i primi, l'uso di frasi modalizzate (nella forma della modalità deontica, come nel noto $V a^{\prime}$ dove ti porta il cuore, di S. Tamaro), o di scelte lessicali attinte ai registri diastraticamente più marcati (si veda, per esempio, il recente successo editoriale, nel filone comico, Anche le formiche, nel loro piccolo, si incazzano, tanto più provocatorio in quanto uscito per i tipi di un editore come Einaudi, solitamente legato a volumi di prestigio scientifico e culturale e ben lontano dalle lusinghe del mercato delle grandi cifre). ${ }^{17}$ In altri casi, le figure retoriche servono a richiamare l'attenzione, giocando con richiami antitetici non sempre collegati direttamente al contenuto: Presto che è tardi! si intitola un volumetto umoristico di E. Greggio (Mondadori), destinato alla satira o, forse meglio, alla caricatura parodistica dei più noti personaggi pubblici dell'Italia contemporanea. E gli esempi potrebbero moltiplicarsi.

Il cosiddetto epitesto allografo prevede almeno due livelli di lancio commerciale:

a. l'intervista: mette l'autore in primo piano e relega la figura del critico letterario in una posizione secondaria;

b. il dibattito: se la promozione è affidata a questa formula, si associa anche ad intenti culturali che travalicano le finalità 'di cassetta'; si pensi alla costituzione delle 'associazioni dei lettori', ormai comuni e numerose anche in provincia, oppure alla abitudine di organizzare 'incontri con l'autore', inaugurata dalle librerie più importanti delle grandi città ed estesasi, ben presto, anche nei centri più piccoli.

L'informazione mediale tende a privilegiare gli strumenti epitestuali e a favorire il contatto diretto fra i nodi terminali del circuito informativo: l'autore e il pubblico.

Quando si parla di pubblico si allude implicitamente all'equazione: insieme dei lettori = insieme degli acquirenti. Nella realtà, i due insiemi non sembrano affatto sovrapponibili ed è sempre possibile che parte del pubblico (ossia di quanti percepiscono, in qualche modo, notizia dell'esistenza di un libro ed informazioni, per quanto generiche, sul suo contenuto) partecipi alla ricezione, senza avere necessariamente letto il testo, approfittando magari dei canali indiretti sovracitati (le interviste, i dibattiti televisivi, o le note di recensione). Nella stessa misura potrà accadere che l'acquisto di un libro non implichi, in rapporto di causa effetto, di diventarne poi lettore. Genette (74) propone opportunamente una divisione funzionale fra lettura e ricezione: si tratta di operazioni distinte che possono ma non debbono necessariamente coincidere. La scissione delle due modalità percettive fa da corollario ad un 
principio dimostrabile nello studio della diffusione del libro, almeno nella sua veste cartacea tradizionale: circolazione di testi non significa sempre e comunque circolazione del pensiero o dei contenuti (Giannini 220 sgg.), proprio perché possedere un libro non significa averlo letto. Il pubblico, inteso in questa accezione genettiana, si presenta, pertanto, come un insieme più vasto dell 'insieme dei lettori e il mercato editoriale moderno punta a stimolare più da vicino l'attenzione di chi compra, rispetto a quella di chi legge: ciò spiega il potenziamento degli effetti paratestuali. L'obiettivo primario dell'inserimento di un libro sul mercato è che sia venduto il più possibile e non che sia letto nelle stesse proporzioni. Da ciò derivano tutte le strategie informative interne ed esterne che ne contrassegnano le sorti editoriali e commerciali.

Nel concludere questa conversazione sulle condizioni dell'informazione sul libro e sugli inevitabili cambiamenti nei criteri di trasmissione e ricezione testuale, ricordo l'osservazione della Bachmann, secondo cui i successi variabili nel tempo di opere e autori danno informazioni sul tempo che li accoglie e li promuove. Essa si fonda sulla convinzione che la letteratura non sia un fatto compiuto, ma un territorio aperto in cui il passato si riversa inevitabilmente sul presente. Parafrasando la forma e la sostanza del pensiero della Bachmann, possiamo dire che anche gli strumenti che le singole epoche selezionano per garantire la circolazione ed il successo di opere e autori ci informano sullo 'spirito dei tempi' medesimi. E francamente mi pare che l'enfasi sul protagonismo dell'autore e sulla spendibilita commerciale della sua immagine non depongano troppo a favore dell'epoca corrente.

\section{Universitd per Stranieri di Perugia}

\section{NOTE}

1 È il testo di una relazione presentata al seminario di studi su "ll libro nella società multimediale", tenutosi presso l'Università per Stranieri di Perugia nel dicembre 1995.

2 L'espressione catene di trasmissione è riferita in Doležel alla circolazione perpetua dei testi letterari: essa rappresenta il "requisito essenziale" per la loro sopravvivenza, poiché "i testi letterari esistono come oggetti estetici solo fintantoché essi vengano attivamente elaborati nella circolazione" (Dolě̌el 210).

3 Il Circolo di Praga (fra i nomi più rappresentativi ricordo: N.S. Trubeckoj, R. Jakobson) è noto al pubblico dei linguisti per i fondamentali contributi alle scienze del linguaggio nei diversi settori (dalla fonologia alla semantica) e alla diffusione dello strutturalismo nel pensiero linguistico e nella cultura europea. Ma altrettanto preziose sono state le indicazioni che i praghesi hanno dato nel campo della critica letteraria e della poetica: l'analisi e la valutazione di questo filone di studi è contenuta in Doleźel (185-220), dove si ricorda come già R. Jakobson riconoscesse la natura composita delle teorie linguistiche e letterarie espresse nel pensiero praghese, autentico punto di incontro delle esperienze scientifiche europee e americane.

4 Scritto e parlato si contrappongono per differenti proprietà legate alla natura stessa del mezzo di trasmissione del messaggio (catene di grafemi e di fonemi, rispettivamente). 1 tratti oppositivi delle varietà diamesiche di base possono essere racchiusi nel seguente schema, che riassume le strategie comunicative di entrambe: 


\begin{tabular}{|c|c|c|c|}
\hline $\begin{array}{l}\text { a. } \\
\text { b. } \\
\text { c. }\end{array}$ & $\begin{array}{l}\text { parlato } \\
\text { dialogicità } \\
\text { dipendenza dai fattori contestuali } \\
\text { dinamismo testuale }\end{array}$ & vs. & $\begin{array}{l}\text { scritto } \\
\text { unidirezionalità } \\
\text { autonomia } \\
\text { fissità }\end{array}$ \\
\hline
\end{tabular}

5 Il dibattito sui criteri di composizione del canone in letteratura è quanto mai vivo ed acceso in questi tempi, nell'ambito della critica epistemologica e letteraria nordamericana. La proposta più radicale, maturata in seno al cosiddetto Cultural Materialism, prevede "l'abolizione radicale della nozione di canone in quanto, qualora si accetti la nozione di costruzione discorsiva della realtà - e dunque la divisıone deı saperi come operazione ștoricamente e culturalmente determinata - non sarà più possibile, né utile, isolare il corpus di testi tradizionalemnte etichettato come letteratura da tutte le altre forme di testualità" (Iuli 170-71).

6 I cultural studies (CS) sono ormai identificabili nella realtà culturale statunitense come un'area di studio in cui convergono una molteplicità di interessi disciplinari, destinati a fomire nuove interpretazioni per temi 'antichi' (il rapporto società/cultura, letteratura/cultura etc.). Il progetto dei $C S$, tuttora in stato di elaborazione a circa un decennio dal suo atto di nascita, ambisce anche a ridefinire il ruolo della cultura di massa e delle strategie di ricezione e consumo dei prodotti culturali di varia natura (da quelli musicali e televisivi, a quelli tradizionalmente etichettabili come letterari). Per una rassegna recente sull'argomento, si veda luli.

7 L'abitudine di consegnare all'editore il testo pronto per la stampa (camera ready-copy direttamente riproducibile su pellicola) è ormai invalsa in Italia nella saggistica e, in modo particolare, in quella accademica. 1 fatti che giustificano questa trasformazione sono in parte noti: spesso i saggi specialistici hanno un pubblico molto circoscritto e, per l'elevato grado di tecnicismo che li caratterizza, raggiungono con difficoltà porzioni più ampie di mercato. Le case editrici adottano, pertanto, una politica commerciale molto cauta: tiratura del volume limitata ad un numero esiguo di copie (sull'ordine di qualche centinaia), contributi finanziari che ne coprano, almeno in parte, i costi vivi, investimento minimo nel lancio commerciale. I rischi di questa circolazione autarchica della stampa scientifica sono stati compresi ed anche, più volte, denunciati (si pensi solo ai toni secchi con cui si è espresso di recente l'italianista Marco Santagata sulle colonne di un noto quotidiano, in merito alle condizioni dell'italianistica). Meno spesso si è riflettuto sul fatto che l' off-set, se ben sfruttato, offre la possibilità di superare i vincoli commerciali: con le suddette modalità 'artigianali' un libro di qualità, ma che magari tratta questioni estranee ai percorsi culturali imposti dai media, potrà essere stampato anche se non obbedisce agli imperativi del marketing editoriale. D'altra parte, il fatto che il suo contenuto scientifico non raggiunga il grande pubblico può significare che i temi affrontati non appartengono alla moda culturale del momento e non, necessariamente, che il suo valore sia modesto. Il Leopardi delle Operette Morali (nel celebre Dialogo di Tristano e di un amico) aveva ben descritto il rapporto fra la qualità del pensiero profuso dagli intellettuali nei libri dell'epoca e la diffusione che esso aveva raggiunto nella comunità. Certe osservazioni mantengono drammaticamente la loro freschezza in riferimento all'Italia dei nostri tempi.

8 Si ricordi, peraltro, che la lettura silenziosa non è invenzione del tardo impero, ma ha radici che risalgono almeno fino al $\mathrm{V}$ a.C. (Svenbro 21).

9 Adotto qui, con facile analogia con i ben noti tecnicismi della sociolinguistica, una nuova etichetta, per cogliere le particolarità formali e sostanziali della lingua trasmessa. Le varietà sociolinguistiche di base sono infatti: $\mathrm{v}$. diatopica, $\mathrm{v}$. diastratica, $\mathrm{v}$. diafasica, $\mathrm{v}$. diamesica, con riferimento rispettivamente agli assi di variazione geografico, sociale, situazionale e legato al mezzo di trasmissione. Anche se può sembrare altrettanto legittima la classificazione all'intemo dell'asse diamesico, poiché i tratti che lo connotano sono legati alla pluralità dei mezzi di trasmissione (mediali e informatici), resta vero che le proprietà comunicative del trasmesso sono diverse sia da quelle dello scritto che da quelle del parlato e ciò costringe a rivedere, sul piano teorico, i confini e l'interazione delle varietà sociolinguistiche, per valutare il corretto inserimento della diatelìa nell' architettura dell'italiano contemporaneo.

10 Per questi ed altri aspetti della 'rivoluzione comunicativa' prodotta dalla diffusione dei mezzi informatici si veda il recente volume di Bettetini. 
11 Senza tradire la comprovata abilità nella suddivisione e nella denominazione delle differenti porzioni del testo, Genette ( $7 \mathrm{sgg}$.) identifica le due dimensioni che compongono il paratesto: il peritesto, categoria spaziale che comprende tutte le indicazioni e i messaggi inseriti fisicamente negli spazi periferici (i cosiddetti 'dintomi': titoli, prefazione, note) e l'epitesto, che include invece tutte le informazioni collocate all'estemo (interviste, conversazioni, corrispondenza etc.). L'unione delle due categorie dà vita al paratesto.

1211 paratesto fattuale viene definito nel modo seguente da Genette: "Qualifico come fattuale un paratesto costituito non da un messaggio esplicito (verbale o altro), ma da un fatto la cui sola esistenza, se conosciuta al pubblico, apporti qualche commento al testo e abbia un peso sulla sua ricezione" (9). Anche il sesso dell' autore rientra in quest'ambito: senz'altro, osserva Genette, non leggiamo con gli stessi occhi la scrittura femminile e quella maschile. Gender e women studies, in gran voga nell'accademia nordamericana a partire dagli inizi di questo decennio, nascono, pertanto, sul terreno epistemologico del migliore strutturalismo europeo.

13 Le considerazioni qui svolte riguardano più direttamente il testo letterario, in quanto nella saggistica l'autore non è solitamente sentito in simbiosi col testo (non si parla, infatti, di "patemità') e la stessa natura del rapporto autore-pubblico è mediata dai diversi fini comunicativi che il testo scientifico possiede.

14 Nelle edizioni di Adelphi, in realtà, sovracoperta e copertina sono tutt'uno nel dorso, mentre sul davanti del libro la sovracoperta si stacca da una copertina volutamente dimessa, realizzata in cartoncino bianco.

15 Alcuni esempi: la raccolta di saggi di Bachmann, oppure il libro-reportage di Chatwin, Adelphi etc.

16 Le tre funzioni fondamentali riconosciute al titolo sono: 1. funzione 'deittica' o 'identificativa'; 2. funzione 'referenziale'; 3. funzione 'espressiva'. L'evoluzione delle tecniche di titolatura ha portato ad una assegnazione, sia pure tendenziale, delle prime due funzioni al sottotitolo. Questa tipologia risale a quella che Genette (75-76) segnala come 'vulgata' funzionale sui compiti informativi del titolo. Manca, a tutt'oggi, uno studio esauriente ed aggiomato sulle funzioni linguistiche del titolo all'intemo del processo informativo e promozionale che accompagna il testo, a partire dai suoi "dintomi".

17 Sarebbe interessante studiare la tipologia dei cataloghi editoriali anche in rapporto alla politica culturale delle case editrici: innovazione e conservatività nella elaborazione dei titoli dovrebbero essere valutate, infatti, tenendo conto del target che l'editore intende raggiungere con i propri volumi e del genere letterario o saggistico privilegiato.

\section{OPERE CITATE}

Bachmann, lngeborg. Letteratura come utopia. Lezioni di Francoforte. Milano: Adelphi, 1993.

Bettetini, Gianfranco. L'audiovisivo. Dal cinema ai nuovi media, Milano: Bompiani, 1996.

Cavallo, Guglielmo, e Roger Chartier, eds. Storia della lettura nel mondo occidentale. Bari: Laterza, 1995.

Chatwin, Bruce. Che ci faccio qui?. Milano: Adelphi, 1989.

Doležel, Lubomír. Occidental Poetics. Tradition and Progress. Lincoln-London: Nebraska UP, 1990. (Trad. it. Poetica occidentale. Tradizione e progresso. Torino: Einaudi, 1990).

Genette, Gérard. Soglie. I dintorni del testo. Torino: Einaudi, 1989.

Giannini, Stefania. Percorsi metalinguistici. Giuliano di Toledo e la teoria della grammatica. Milano: Angeli, 1996.

Handke, Peter. Intervista sulla scrittura. Bergamo: Pierluigi Lubrina Editore, 1990.

Iuli, Maria Cristina. "I Cultural Studies". Donatella Izzo, ed. Teoria della letteratura. Prospettive dagli Stati Uniti. Roma: La Nuova Italia Scientifica, 1996. 159-84.

Svenbro, Jesper. "La Grecia arcaica e classica: l'invenzione della lettura silenziosa". Cavallo e Chartier 3-36.

Wittmann, Reinhard. "Una «rivoluzione della lettura» alla fine del XVIII secolo?". Cavallo e Chartier 337-69. 